\title{
Nuclear Black Hole Formation in Clumpy Galaxies at High Redshift
}

\author{
Bruce G. Elmegreen \\ IBM Research Division, T.J. Watson Research Center, P.O. Box 218, Yorktown Heights, \\ NY 10598, USA \\ bge@watson.ibm.com \\ Frédéric Bournaud \\ Laboratoire AIM, CEA-Saclay DSM/IRFU/SAP - CNRS - Université Paris Diderot, \\ F-91191 Gif-sur-Yvette Cedex, France \\ frederic.bournaud@cea.fr \\ Debra Meloy Elmegreen \\ Vassar College, Dept. of Physics 85 Astronomy, Box 745, Poughkeepsie, NY 12604 \\ elmegreen@vassar.edu
}

\begin{abstract}
Massive stellar clumps in high redshift galaxies interact and migrate to the center to form a bulge and exponential disk in $\lesssim 1$ Gyr. Here we consider the fate of intermediate mass black holes (BHs) that might form by massive-star coalescence in the dense young clusters of these disk clumps. We find that the BHs move inward with the clumps and reach the inner few hundred parsecs in only a few orbit times. There they could merge into a supermassive BH by dynamical friction. The ratio of $\mathrm{BH}$ mass to stellar mass in the disk clumps is approximately preserved in the final ratio of $\mathrm{BH}$ to bulge mass. Because this ratio for individual clusters has been estimated to be $\sim 10^{-3}$, the observed $\mathrm{BH}-$ to-bulge mass ratio results. We also obtain a relation between $\mathrm{BH}$ mass and bulge velocity dispersion that is compatible with observations of present-day galaxies.
\end{abstract}

Subject headings: instabilities — stellar dynamics — galaxies: bulges — galaxies: clusters - black holes 


\section{Introduction}

Numerical simulations have reproduced the massive clumpy structures of star formation in high-redshift galaxies and followed the migration of these clumps into the galaxy centers where they merge to form bulges (Noguchi 1999; Immeli 2004ab; for a review of clumpy structures, see Elmegreen 2007). The clumps result from gravitational instabilities in a gas-rich, highly-turbulent disk, and the central migration results from clump interactions and angular momentum losses to the disk, halo, and clump debris. In a series of papers, we have shown that the resulting disk has the characteristic double-exponential profile of modern spiral galaxies (Bournaud, Elmegreen \& Elmegreen 2007, hereafter BEE07), and the bulge has a classical form, with high Sersic index, three-dimensional random motions, little rotation, and a rapid formation (Elmegreen, Bournaud, \& Elmegreen 2008; hereafter EBE08). We have also reproduced in detail the peculiar morphology and kinematics of a galaxy in the Hubble Space Telescope Ultra Deep Field, UDF 6462, with this model (Bournaud et al. 2008). Other spectroscopic observations also indicate, less directly but over a larger sample, that high-redshift disk and bulge evolution is characterized by giant clump interactions and high turbulence (e.g., Förster Schreiber 2006), which is consistent with our models.

This paper considers another aspect of clumpy disk evolution, the formation of nuclear black holes $(\mathrm{BHs})$. Models of bulge formation should be able to explain how BHs form at the same time, why the BH-to-bulge mass ratio is $\simeq 0.002$ (McLure \& Dunlop 2002; Marconi \& Hunt 2003), and why the BH mass and bulge central velocity dispersion are related by $\log \left(M_{B H} / M_{\odot}\right)=8.13+4.02 \log \left(\sigma_{\text {bulge }} / 200 \mathrm{~km} \mathrm{~s}^{-1}\right)$ (Ferrarese \& Merritt 2000; Tremaine et al. 2002). Here we model all of these observations by considering that each clump forms an intermediate mass black hole (IMBH) by stellar coalescence. We follow the evolution of these IMBHs as their clumps move in the disk. We find that the IMBHs migrate inward along with the clumps and that the final central BH-to-bulge mass ratio is approximately the same as the initial BH-to-clump ratio. This is about the observed value for bulges. The velocity dispersion relation for BHs in bulges also results to a reasonable approximation.

In the following, Section 2 outlines our model for nuclear BH formation, section 3 describes the numerical simulations, section 4 gives the results, and section 5 contains a brief discussion. 


\section{Black Hole Formation Model}

Nuclear BHs are an important aspect of galaxy and bulge formation. Malbon et al. (2007) summarized BH models by suggesting that gas accretion during starbursts forms relatively low mass $\mathrm{BH}$ s at high redshift, while $\mathrm{BH}$ coalescence during galaxy mergers forms supermassive BHs at low redshift (see also works by Di Matteo et al. 2005, 2007; Johansson, Naab, \& Burkert 2008). Disk density waves are less efficient in fueling nuclear black holes (Younger et al. 2008). In a very different type of model, Ebisuzaki et al. (2001) suggested that IMBHs grow by stellar coalescence in dense young clusters that form in the central regions of galaxies. Dynamical friction then forces these IMBHs to the center where they merge into a nuclear BH. Here we determine whether a model like this can also apply to IMBHs that form in dense disk clusters, far from the nucleus. We know from clumpy disk models that the disk clusters migrate to the center to form a bulge, so the primary question here is whether IMBHs that form in these clusters follow them inward to the nucleus. The BH-to-cluster mass ratio was found in the simulations by Ebisuzaki et al. (2001) to be $\sim 10^{-3}$, which is the same as the BH-to-bulge mass ratio. Thus, what we primarily need to determine is whether this mass fraction is preserved during the clump/BH migration. There are two important differences from the Ebisuzaki et al. model: (1) the clusters here are much more massive than they considered, so the IMBHs are more massive also $\left(\sim 10^{5} M_{\odot}\right.$ each in our models), and (2) our clusters start in the main galaxy disk rather than the central regions, so they come in as a result of gravitational interactions between clumps, not dynamical friction. We also assume that the giant clumps observed in high redshift disks are composed of denser, unresolved clusters, where the stars actually form, just as star complexes in local galaxies form most of their stars in dense clusters. The IMBHs presumably form inside these dense clusters.

Cluster simulations have generally supported this model for IMBH formation. PortegiesZwart \& McMillan (2002) confirmed that runaway stellar collisions can make cluster BHs with $10^{-3}$ of the cluster mass during core collapse if the relaxation time is less than $25 \mathrm{Myr}$. Gürkan, Freitag \& Rasio (2004) did a cluster simulation with $10^{7}$ stars and found mass segregation and core collapse in less than an O-star lifetime, at which point an IMBH with $10^{-3}$ of the cluster mass formed. Portegies-Zwart et al. (2004) applied the model to the suspected IMBH in M82 (Matsumoto et al. 2001). Gürkan, Fregeau, \& Rasio (2006) showed that stellar collisions in clusters with binaries could make two IMBHs which form their own binary. Freitag, Gürkan \& Rasio (2006) included stellar collisions in a cluster simulation and found that an IMBH forms when core collapse happens faster than the main sequence lifetime of a massive star; this requires densities of $10^{6}-10^{7} \mathrm{pc}^{-3}$ (Freitag 2007). For the Milky Way nucleus, Portegies-Zwart et al. (2006) showed that $10 \%$ of dense clusters form IMBHs during their inward migration and they coalesce fast enough to explain the massive 
BH now. Matsubayashi, Makino, \& Ebisuzaki (2007) found that dynamical friction on IMBHs becomes ineffective close to the central BH, but the IMBH spirals in anyway because of gravitational radiation. These theoretical studies suggest that at least some nuclear BHs could have formed by the merger of IMBHs from dense massive clusters. Subsequent gas accretion would presumably have caused the BHs to grow to their present masses. Feedback effects (e.g., McLaughlin et al. 2006) might be important at this later gas accretion stage.

\section{Numerical Simulations}

The evolution of gas-rich galaxy disks is modeled with a particle-mesh sticky-particle code (Bournaud \& Combes 2002, 2003) with a grid resolution and gravitational softening length of 110 pc. Stars, gas, and dark matter halo are each modeled with one million particles. The sticky-particle parameters are $\beta_{r}=\beta_{t}=0.7$ for all but run 4 , which has $\beta_{r}=\beta_{t}=0.8$. We assume a local Schmidt law for star formation in which the probability per timestep that each gas particle is transformed into a stellar particle is proportional to the 1.4 power of the local gas density (Kennicutt 1998). The proportionality factor gives a star formation rate of $3.5 \mathrm{M}_{\odot} \mathrm{yr}^{-1}$ in the initial disk. Star formation feedback is not expected to be important for the formation and lifetime of the clumps because of their enormous masses and deep local potential wells (BEE07).

The initial model parameters were summarized in Table 1 of BEE07; runs 1-7 are the same here except for the addition of BHs. We review the assumptions briefly here. The initial disk is composed of gas and stars with a uniform surface density. This follows from the observation that extremely clumpy disks at $z \geq 1$ do not have exponential profiles (Elmegreen et al. 2005). The initial disk radius is $6 \mathrm{kpc}$ (typical for clump cluster, chain, and spiral galaxies at $z \geq 1$ - Elmegreen et al. 2007a) and the initial thickness is $h=700 \mathrm{pc}$

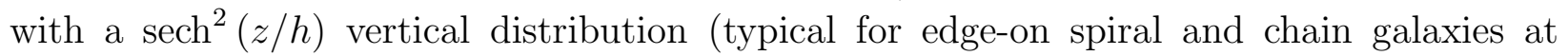
$z \geq 1$ - Elmegreen \& Elmegreen 2006). The disk mass is $7 \times 10^{10} \mathrm{M}_{\odot}$. Stars have a Toomre parameter in the stable regime, $Q_{\mathrm{s}}=1.5$. The initial velocity dispersion of the gas, $\sigma_{\text {gas }}$, is $9 \mathrm{~km} \mathrm{~s}^{-1}$ for all runs but 1,2 , and 3 , where it is 5,15 , and $20 \mathrm{~km} \mathrm{~s}^{-1}$, respectively. The gas mass fraction in the disk, $f_{\mathrm{G}}$, is 0.5 except for runs 4 and 5 , where it is 0.25 and 0.75 . The halo-to-disk mass ratio, $H / D$, inside the initial disk radius, is 0.5 except for runs 6 and 7 , where it is 0.25 and 0.80 . The small number of massive clumps observed in high-redshift disks implies a relatively high ratio of turbulent speed to rotation speed, $\sim 10 \%$ or more. To get such a turbulent disk gravitationally unstable so that it forms clumps, we need a fairly high gas column density, which means a high gas-to-star ratio in the disk at that time (see BEE07, EBE08). 
We have performed simulations for our series of studies that have three types of initial halo and bulge properties: runs 0 to 7 have no initial bulge and a dark halo that is a Plummer sphere with a scale-length of $15 \mathrm{kpc}$. Runs $0 \mathrm{~N}, 1 \mathrm{~N}$, and $2 \mathrm{~N}$ have a $\Lambda$ CDM cuspy halo (Navarro, Frenk \& White 1997) with a cusp scale-length $r_{S}=6 \mathrm{kpc}$ (concentration parameter 16.7 for a virial radius of $100 \mathrm{kpc}$ ). Runs $0 \mathrm{~B}, 1 \mathrm{~B}$, and $2 \mathrm{~B}$ have a small initial bulge that is a Plummer sphere with $10 \%$ of the disk mass and a radial scale-length of 600 pc. The other parameters for these six runs, including the gas fractions and halo-to-disk mass ratios, are unchanged from runs 0,1 , and 2 , respectively.

Massive clumps form quickly in all simulations, in about the local dynamical time at the midplane gas density. Their masses and sizes are comparable to the local Jeans mass and size. The clumps were identified objectively every $25 \mathrm{Myr}$ as regions where the surface density is locally larger than the radial average by a factor of 3 . Only clumps with masses larger than $2 \times 10^{8} M_{\odot}$ and sizes smaller than $3 \mathrm{kpc}$ were considered to be clumps (this avoids misidentifying spiral arms as clumps). When the mass fraction in the clumps reached its maximum value (usually at a time of about $200 \mathrm{Myr}$ in the simulation), single particles representing $\mathrm{BHs}$ were positioned in the centers of all the identified clumps, one for each clump. The particle masses were equal to $10^{-3}$ times the clump masses at that time. They were initially placed at the positions of peak density. To ensure mass conservation, we removed the corresponding number of gas and star particles, randomly chosen within the gravitational softening length. Thereafter, the BH particles were treated like massive star particles that could be moved only by gravity. The $10^{-3} \mathrm{BH}$ fraction in the clumps was fixed

for the reasons detailed above. We do not aim at resolving the processes driving it, like the mass accretion onto the BHs and the potentially associated AGN feedback.

The BH particles have such low masses that they do not affect the clump dynamics, as confirmed by detailed comparisons with runs having no $\mathrm{BH}$ particles. Neither do the $\mathrm{BH}$ particles interact with each other much in the disk environment. If we consider an interaction to be an approach within $200 \mathrm{pc}$, then the number of such interactions at radii greater than $2 \mathrm{kpc}$ in the disk is a total of 6 for all 8 runs and $58 \mathrm{BHs}$ produced in these runs. That is a $10 \%$ effect. An additional 4 total BHs interacted between 1 and $2 \mathrm{kpc}$ radius. By far most of the BHs interact with each other only when they get to the nucleus.

\section{Results}

The time evolution of the gas+star mass column density is shown for run 0 in Figure 1. White dots represent the initial IMBH particles. They form inside the clumps at moderate to large radii in the primordial disk, and then migrate to the center with the clump cores. 
The entire process takes about 1 Gyr. Run 0 models without BHs were shown in BEE07 and run 0 models with cuspy dark matter halos $(0 \mathrm{~N}, 1 \mathrm{~N}, 2 \mathrm{~N})$ and small initial bulges (0B, 1B, 2B) were shown without BHs in EBE08. The clump evolution is indistinguishable when $\mathrm{BHs}$ are included, so we do not repeat the $0 \mathrm{~N}$ and $0 \mathrm{~B}$ figures here in the $\mathrm{BH}$ cases.

Table 1 lists the numbers of BHs that formed and the numbers that get within the central 250, 500, and $1000 \mathrm{pc}$ by the end of each run. It also gives the ratios of the $\mathrm{BH}$ masses that reach the inner 500 pc or 1000 pc to the bulge masses. Some low-mass BHs from low-mass clumps do not reach the central regions, but most BHs reach the central 500 pc and even the central $250 \mathrm{pc}$. The final bulge and BH masses are also tabulated, as are the bulge-to-total mass ratios, the BH-to-bulge mass ratios, and the bulge velocity dispersions. These dispersions, $\sigma_{\text {bulge }}$, are the average line-of-sight values for 502 -D projections of the stars, uniformly distributed over the sine of the inclination angle. Each dispersion comes from all the stars inside a projected aperture diameter of $110 \mathrm{pc}$ (the grid resolution). We do not subtract the disk component from edge-on projections, nor do we make a direct 3-D measurement, because these would not be done in observations.

Figure 2 plots the final versus the initial $\mathrm{BH}$ galactocentric radii to show how common it is for the clump BHs to reach the galaxy centers. Black holes that start in clumps that are far out in the disk do not typically reach the center; they move inward only about a factor of 2 in radius and then the clump disperses without adding to the bulge. The figure also includes simulations that have cuspy halos (circles) and those that have a small initial bulge. In all cases, clumps form in the disk by gravitational instabilities, and BHs that are placed in these clumps migrate inward along with the clumps. Those that start within $\sim 4$ kpc get all the way to the center in 1 Gyr or less. The BHs that get in the furthest are the most massive ones that formed in the most massive clumps (Table 1).

Bulges and centralized BH clusters always form together in our models. The total mass of the centralized BHs (those reaching the central $500 \mathrm{pc}$ ) is usually within a factor of 2 of the initial BH mass formed in the clumps. The IMBHs that do not reach the central regions (those from the smallest clumps) represent a small fraction of the total $\mathrm{BH}$ mass. The $\mathrm{BH} /$ bulge mass fraction can therefore be written,

$$
\frac{M_{B H}}{M_{\text {bulge }}} \sim\left(\frac{M_{B H}}{M_{\text {clump }}}\right)\left(\frac{M_{\text {clump }}}{M_{\text {bulge,clumps }}}\right)\left(\frac{M_{\text {bulge,clumps }}}{M_{\text {bulge }}}\right)
$$

where $M_{B H} / M_{\text {clump }}=10^{-3}$ according to cluster simulations. In this equation, $M_{\text {clump }}$ is the total clump mass, $M_{\text {bulge,clump }}$ is the mass in the bulge that comes from the clumps, and $M_{b u l g e}$ is the total bulge mass. Typically $M_{\text {clump }} / M_{\text {bulge,clumps }} \sim 2$ and $M_{\text {bulge,clumps }} / M_{\text {bulge }} \sim 0.5$ (see BEE07). Note that half of the bulge stars come from intense star formation in the bulge region during the clump merging process. This makes up for the half of the clump stars that 
are left behind in the disk. Gas accretion onto the BHs should increase their mass after they get to the nucleus, and other processes could add to the bulge and disk later too.

Figure 3 shows the final nuclear BH mass, taken from the mass of IMBHs that migrated within radii $<500 \mathrm{pc}$, versus the final bulge line-of-sight velocity dispersion. There is a clear correlation. The dashed line has a slope of 4 , which is the observed value, and the solid line has a slope of 2 , which is a reasonable approximation to the lower envelope. The absolute scale depends on uncertain assumptions about the initial value of $M_{B H} / M_{c l u m p}$, and on whether we assume nuclear BHs come from IMBHs within 250 pc or $1000 \mathrm{kpc}$ (see Table 1). For a dispersion of $130 \mathrm{~km} \mathrm{~s}^{-1}$, which is in the middle of the plot, the Tremaine et al. (2002) $\mathrm{BH}$ mass would be $2.4 \times 10^{7} M_{\odot}$, whereas we get half this value, $1.3 \times 10^{7} M_{\odot}$. We consider the existence of a correlation to suggest that the proposed model is plausible. The model details are too crude and the bulge mass range considered is too small to be more conclusive at this time. For example, the $\mathrm{BH} /$ clump mass fraction, assumed to be a constant $10^{-3}$, could be higher in higher mass clumps or in more massive galaxies; this could change the correlation slightly. Other processes are likely to contribute to the bulge dispersions and the BH masses too, and they could alter the slope. For example, AGN feedback (neglected in our simulations) could bring the slope of the BH mass - velocity dispersion relation closer to the presently observed value (McLaughlin et al. 2006) if BH formation occurs quickly, as in our present model. Observations at high redshift suggest that the correlation evolves (Peng et al. 2006ab; Treu et al. 2007; Woo et al. 2008).

\section{Discussion}

Numerical simulations suggest that primitive gas-rich disks should fragment into clumpy star-forming complexes with masses of $\sim 10^{8} M_{\odot}$ or more, and that these complexes should interact gravitationally and move to the galaxy center where they combine to form a bulge. The surrounding disk becomes exponential in the process. If the complexes contain massive dense clusters, and if these clusters form IMBHs, then the IMBHs will migrate to the center too. Our model assumes that the centralized BHs eventually merge into a single, nuclear $\mathrm{BH}$. This assumption makes our model significantly different than $\mathrm{BH}$ formation models that rely on massive gas accretion to feed a seed nuclear $\mathrm{BH}$.

Relativistic effects during BH mergers cannot be treated by our model. Even their capture into $\mathrm{BH}$ binaries as a first step toward merging cannot be simulated, because the gravity softening length is comparable to their final separation. Still, BH binaries should form because of strong dynamical friction in a gas-rich environment (Escala et al. 2005). This process has been resolved in models by Mayer et al. (2007). Gravitational radiation should 
then drive their eventual merger. However, coalescing BHs can experience velocity kicks from the anisotropy of gravity waves, and these velocity kicks can be as large as hundreds or even thousands of $\mathrm{km} \mathrm{s}^{-1}$ (e.g., Baker et al. 2007). Such fast-moving BHs could be ejected from the galaxy (Merritt et al. 2004). This would challenge many models of nuclear $\mathrm{BH}$ formation, including the common idea that they grow from mergers during the hierarchical galaxy build-up (e.g., Di Matteo et al. 2007).

A solution to this problem was proposed by Bogdanovic et al. (2007) in the hierarchical merging context. According to Baker et al. (2007), the kick is greatly reduced if the merging BHs have spins aligned with each other. Bogdanovic et al. (2007) showed that such alignments can result from torques in gas-rich merging galaxies. In the present scenario where disk clumps merge together, gas torques may contribute in the same way, but it is also likely that the BHs will have their spins already aligned with their orbit angular momenta. This is because the clumps in which they form are all fragments of the same initial galaxy disk; they have the same spin orientation and the same orbital angular momentum when they coalesce (BEE07; Bournaud et al. 2008). The BHs should preserve these alignments and coalesce without experiencing major kicks. Even clumps that merge outside the central kpc should have their spins aligned, again reducing the chance for velocity kicks in BHs that merge. We also note that recoiling BHs would settle back rapidly to the galaxy center, as long as the velocity kick does not exceed the escape velocity (Blecha \& Loeb 2008).

Both classical and pseudo-bulges have nuclear BHs with masses proportional to the bulge mass (Kormendy \& Richstone 1995; Magorrian et al. 1998) and a power of the velocity dispersion (Ferrarese \& Merritt 2000; Gebhardt et al. 2000; Novak et al. 2006). Active galaxies follow the same relation as non-active galaxies (Nelson 2000). These correlations may change slightly over time. At intermediate redshifts, $z \sim 0.36-1$, the bulge mass may be $\sim 2 \times$ smaller than the modern bulge mass compared to the BH mass (Treu et al. 2007), and at higher redshifts, $z>1.7$, the bulge may be $\sim 4 \times$ smaller (Peng et al. 2006ab). Thus bulges seem to grow for a slightly longer time than BHs. Salviander et al. (2007) and Lauer et al. (2007) caution that some of this appearance of late bulge growth may result from observational bias in favor of more active nuclei. In our model the time evolution may be explained by continued bulge growth during minor mergers and secular evolution, after the first bulge and its $\mathrm{BH}$ formed via clump/IMBH coalescence. If it takes a highly gas-rich disk to make clusters dense enough to form IMBHs, then only the first generation of clumps will add to the nuclear $\mathrm{BH}$ and the rest may add only to the bulge.

Small-bulge galaxies $\left(M<10^{10} M_{\odot}\right)$ have compact nuclear star clusters instead of BHs (e.g., Carollo et al. 1998; Matthews et al. 1999; Böker et al. 2002; 2004). Massive galaxies can have dense nuclear clusters too (Seth et al. 2008). These clusters have about the same 
correlations with bulge mass and velocity dispersion as the BHs (Wehner \& Harris 2006; Rossa et al. 2006; Graham \& Driver 2007; Ferrarese et al. 2006; Côté et al. 2006; Li et al. 2007a), but somehow the dense gas, whether in disk clumps or in the nuclei themselves, made stars instead of BHs, or they made stars that could not coalesce into BHs. In our model, this difference is mostly the result of a difference in disk-clump density, with high density clumps more likely to form IMBHs inside their dense cores and low density clumps forming only dense stellar clusters. Dense clusters should migrate to the galaxy centers in the same way as BHs if the clusters do not evaporate first. This is consistent with the observation that nuclear clusters typically contain a range of ages and are on average younger than their disks (Rossa et al. 2006; Côté et al. 2006). We suggest that galaxies with the highest disk gas fractions and the highest disk turbulent speeds make the densest and most massive disk clumps, and that these are the formation sites for IMBHs. Less extreme disks, or later stages of the same disks, make only dense star clusters in their disk clumps.

The observed correlation between galaxy mass and the presence or lack of nuclear BHs then follows from the correlation between galaxy mass and density: higher mass galaxies are generally denser and should form denser clumps and IMBHs in those clumps, while lower mass galaxies have lower density disks and should form lower-density clumps that do not make IMBHs. Both types of clumps will be relatively massive compared to their disks (because both types of galaxies presumably have high ratios of turbulent to rotational speeds at early times) and therefore both types of clumps will interact and migrate to the center as we simulate here. However, only the dense clumps in massive galaxies will bring IMBHs to the nucleus. It is also possible that nuclear BHs form earlier in the life of a massive galaxy than nuclear star clusters form in the life of a low-mass galaxies, because of the shorter dynamical time for the massive galaxy. In that case, nuclear clusters could take much longer to form than nuclear BHs, when measured in absolute time. Galaxy interactions should also increase the turbulent speed and disk gas density, promoting the formation of IMBHs. In that case, tidal torques as well as clump interactions would bring clump stars and IMBHs into the center.

Dense nuclear clusters would not be expected to form IMBHs in the same way as dense young clusters if the nuclear clusters assembled from in-spiraling evolved clusters, which have no massive stars anymore. Low mass stars are small and have much smaller gravitational cross sections than high mass stars, and mass segregation in a young cluster puts the high mass stars close together where they can interact quickly. Thus it is reasonable that young clusters in dense disk clumps make IMBHs, which spiral in to the center to make nuclear BHs, while neither young clusters in low-density disks nor evolved cluster debris in galactic nuclei can make BHs by the same mechanism, which is direct stellar collisions. 
Our model applies primarily to BH formation at high redshift, which occurs in galaxies that should be gas-rich and highly turbulent - the two primary ingredients for the evolution found here. Our model also forms BHs rapidly and in a gas-rich environment, which triggers an intense starburst. These aspects of BH formation are in agreement with observations (e.g., Alexander et al. 2005; Escala 2006; Haiman, Jimenez, \& Bernardi 2007). Supermassive BHs in $z \sim 6$ quasars (Fan 2006) could also be formed by disk clump coalescence, because it takes only a few disk rotations to bring the massive clumps to the center. Galaxy interactions would have made clump migration and BH formation even faster. However, our process would not apply to a starless central region, such as that found by Walter et al. (2004) in a $z=6.42$ QSO; gas-related accretion during mergers would be preferred there (e.g., Li et al. 2007b). Our mechanism also cannot form nuclear BHs without bulges, as observed in some galaxies (e.g., Filippenko \& Ho 2003). Such BHs might have formed in nuclear clusters by stellar coalescence.

Our model predicts IMBH activity in high-redshift disk clumps. Such activity may include X-ray, jet, and radio emission if the self-absorption is not too large. We also predict that lower density and more quiescent disks should make smaller disk clumps and possibly no IMBHs, which would be replaced by clusters with too little density for stellar coalescence. Clumpy galaxies at $z>1$ outnumber starburst spirals and ellipticals by a factor 2 in the UDF (Elmegreen et al. 2007a). Given the short timescale of the clumpy phase in our model ( $\sim 1$ Gyr), all present-day early-type disk galaxies would seem to have gone through a clumpcluster or chain-galaxy phase, forming their bulges this way or adding to a smaller bulge

formed earlier by primordial galaxy mergers. Black hole growth by gas accretion should follow their formation by IMBH coalescence. AGN feedback effects might be important during the gas-accretion stage.

Numerical simulations were carried out on the NEC-SX8R vector computer at CEA/CCRT. D.M.E. thanks Vassar College for publication support. Helpful comments by the referee are appreciated.

\section{REFERENCES}

Alexander, D. M., Smail, I., Bauer, F. E., Chapman, S. C., Blain, A. W., Brandt, W. N., \& Ivison, R. J. 2005, Nature, 434, 738

Baker, J. G., Boggs, W. D., Centrella, J., Kelly, B. J., McWilliams, S. T., Miller, M. C., \& van Meter, J. R. 2007, ApJ, 668, 1140 
Blecha, L., \& Loeb, A. 2008, astro-ph/0805.1420

Bogdanović, T., Reynolds, C. S., \& Miller, M. C. 2007, ApJ, 661, L147

Böker, T., Laine, S., van der Marel, R. P., Sarzi, M., Rix, H.-W., Ho, L., \& Shields, J. C. 2002, AJ, 123, 1389

Böker, T., Sarzi, M., McLaughlin, D. E., van der Marel, R. P., Rix, H.-W., Ho, L. C., \& Shields, J. C. 2004, AJ, 127, 105

Bournaud, F., \& Combes, F. 2002, A\&A, 392, 83

Bournaud, F., \& Combes, F. 2003, A\&A, 401, 817

Bournaud, F., Elmegreen, B.G., \& Elmegreen, D.M. 2007, ApJ, 670, 237

Bournaud, F., Daddi, E., Elmegreen, B. G., Elmegreen, D. M. \& Elbaz, D. 2008, A\&A in press, astroph/0803.3831

Carollo, C. M., Stiavelli, M., \& Mack, J. 1998, AJ, 116, 68

Côté, P., et al. 2006, ApJS, 165, 57

Di Matteo, T., Springel, V., \& Hernquist, L. 2005, Nature, 433, 604

Di Matteo, T., Colberg, J., Springel, V., Hernquist, L., \& Sijacki, D. 2008, ApJ, 676, 33

Ebisuzaki, T. et al. 2001, ApJ, 562, L19

Elmegreen, D.M. 2007, in IAU Symosium 235, ed. F. Combes \& J. Palous, Cambridge: Cambridge Univ. Press, in press.

Elmegreen, B.G., Elmegreen, D.M., Vollbach, D.R., Foster, E.R., \& Ferguson, T.E., 2005, ApJ, 634, 101

Elmegreen, B.G., \& Elmegreen, D.M., 2006, ApJ, 650, 644

Elmegreen, D.M., Elmegreen, B.G., Ravindranath, S., \& Coe, D.A., 2007a, ApJ, 658, 763

Elmegreen, B.G., Bournaud, F., \& Elmegreen, D.M. 2008, ApJ, submitted

Escala, A. 2006, ApJ, 648, L13

Escala, A., Larson, R. B., Coppi, P. S., \& Mardones, D. 2005, ApJ, 630, 152

Fan, X. 2006, MmSAI, 77, 635 
Ferrarese, L., \& Merritt, D. 2000, ApJ, 539, L9

Ferrarese, L., et al. 2006, ApJ, 644, L21

Filippenko, A. V., \& Ho, L. C. 2003, ApJL, 588, L13

Förster Schreiber, N. M., et al. 2006, ApJ, 645, 1062

Freitag, M., Gürkan, M.A., \& Rasio, F.A. 2006, MNRAS, 368, 141

Freitag, M. 2007, astroph/0711.4057

Gebhardt, K., et al. 2000, ApJ, 539, L13

Graham, A.W., \& Driver, S.P. 2007, ApJ, 655, 77

Gürkan, M. A., Freitag, M., \& Rasio, F. A. 2004, ApJ, 604, 632

Gürkan, M. A., Fregeau, J.M., \& Rasio, F.A. 2006, ApJ, 640, L39

Haiman, Z., Jimenez, R., \& Bernardi, M. 2007, ApJ, 658, 721

Immeli, A., Samland, M., Gerhard, O., \& Westera, P. 2004a, A\&A, 413, 547

Immeli, A., Samland, M., Westera, P., \& Gerhard, O. 2004b, ApJ, 611, 20

Johansson, P.H., Naab, T., \& Burkert, A. 2008, ApJ, submitted, astro-ph/0802.0210

Kennicutt, R.C., Jr. 1998, ApJ, 498, 541

Kormendy, J., \& Richstone, D. 1995, ARAA, 33, 581

Lauer, T.R., Tremaine, S., Richstone, D., \& Faber, S. M. 2007, ApJ, 670, 249

Li, Y., et al. 2007a, ApJ, 665, 187

Li, Y., Haiman, Z., \& Mac Low, M. 2007b, ApJ, 663, 61

Malbon, R.K., Baugh, C. M., Frenk, C. S., \& Lacey, C. G. 2007, MNRAS, 382, 1394

McLaughlin, D.E., King, A.R., \& Nayakshin, S. 2006, ApJ, 650, L37

McLure, R.J., \& Dunlop, J.S. 2002, MNRAS, 331, 795

Magorrian, J., Tremaine, S., Richstone, D., Bender, R., Bower, G., Dressler, A., Faber, S. M., Gebhardt, K., Green, R., Grillmair, C., Kormendy, J., \& Lauer, T. 1998, AJ, 115, 2285 
Marconi, A., \& Hunt, L. 2003, ApJ, 589, L21

Matsubayashi, T., Makino, J., \& Ebisuzaki, T. 2007, ApJ, 656, 879

Matsumoto, H., et al. 2001, ApJ, 547, L25

Matthews, L.D., et al. 1999, AJ, 118, 208

Mayer, L., Kazantzidis, S., Madau, P., Colpi, M., Quinn, T., \& Wadsley, J. 2007, Science, 316,1874

Merritt, D., Milosavljević, M., Favata, M., Hughes, S. A., \& Holz, D. E. 2004, ApJ, 607, L9

Navarro, J.F., Frenk, C.S., \& White, S.D.M. 1997, ApJ, 490, 493

Nelson, C.H. 2000, ApJ, 544, L91

Noguchi, M. 1999, ApJ, 514, 77

Noordermeer, E., van der Hulst, J. M., Sancisi, R., Swaters, R. S., \& van Albada, T. S. 2007, MNRAS, 376, 1513

Novak, G.S., Faber, S.M., \& Dekel, A. 2006, ApJ, 637, 96

Peng, C. Y., Impey, C. D., Ho, L. C., Barton, E. J., \& Rix, H.-W. 2006a, ApJ, 640, 114

Peng, C. Y., Impey, C. D., Rix, H.-W., Kochanek, C. S., Keeton, C. R., Falco, E. E., Lehr, J., \& McLeod, B. A. 2006b, ApJ, 649, 616

Pfenniger, D., \& Norman, C. 1990, 363, 391

Portegies-Zwart, S. F., \& McMillan, S. L. W. 2002, ApJ, 576, 899

Portegies-Zwart, S. F., Baumgardt, H., Hut, P., Makino, J., \& McMillan, S. L. W. 2004, Nature, 428, 6984

Portegies-Zwart, S. F., Baumgardt, H., McMillan, S. L. W., Makino, J., Hut, P., \& Ebisuzaki, T. 2006, ApJ, 641, 319

Rossa, J., van der Marel, R.P., Böker, T., Gerssen, J., Ho, L.C., Rix, H.-W., Shields, J.C., \& Walcher, C.-J. 2006, AJ, 132, 1074

Salviander, S., Shields, G. A., Gebhardt, K., \& Bonning, E. W. 2007, ApJ, 662, 131

Seth, A., Agueros, M., Lee, D., \& Basu-Zych, A. 2008. astroph/0801.0439 
Tremaine, S. et al. 2002, ApJ, 574, 740

Treu, T., Woo, J.-H., Malkan, M.A., \& Blandford, R.D. 2007, ApJ, 667, 117

Walter, F., Carilli, C., Bertoldi, F., Menten, K., Cox, P., Lo, K. Y., Fan, X., \& Strauss, M.A. 2004, ApJ, 615, L17

Wehner, E. H., \& Harris, W.E. 2006, ApJ, 644, L14

Woo, J. -H., Treu, T., Malkan, M. A., \& Blandford, R. 2008, astro-ph/0804.0235

Younger, J.D., Hopkins, P.F., Cox, T. J., \& Hernquist, L. 2008, astro-ph/0804.2672 
Table 1: Black Hole Migration and Final Bulge Properties

\begin{tabular}{cccccccccccc}
\hline \hline Run & $N_{i}$ & $N_{250}$ & $N_{500}$ & $N_{1000}$ & $f_{M, 500}$ & $f_{M, 1000}$ & $\begin{array}{c}M_{\text {Bulge }} \\
\times 10^{9} M_{\odot}\end{array}$ & $\begin{array}{c}M_{B H} \\
\times 10^{6} M_{\odot}\end{array}$ & $\mathrm{B} / \mathrm{T}$ & $\begin{array}{c}\mathrm{BH} / \mathrm{B} \\
\left(\times 10^{-4}\right)\end{array}$ & $\begin{array}{c}\sigma_{\text {bulge }} \\
\mathrm{km} \mathrm{s}^{-1}\end{array}$ \\
\hline 0 & 6 & 3 & 4 & 5 & 0.69 & 0.93 & 21 & 11.3 & 0.30 & 5.4 & 121 \\
1 & 7 & 3 & 7 & 7 & 1.00 & 1.00 & 14.7 & 9.7 & 0.21 & 6.6 & 117 \\
2 & 7 & 2 & 5 & 6 & 0.78 & 0.92 & 13.3 & 6.4 & 0.19 & 4.8 & 108 \\
3 & 7 & 3 & 6 & 7 & 0.86 & 1.00 & 23.1 & 16.8 & 0.33 & 7.3 & 146 \\
4 & 6 & 2 & 3 & 5 & 0.61 & 0.83 & 9.8 & 3.2 & 0.14 & 3.3 & 87 \\
5 & 6 & 2 & 5 & 5 & 0.91 & 0.91 & 22.4 & 19.2 & 0.32 & 8.6 & 184 \\
6 & 5 & 3 & 5 & 5 & 1.00 & 1.00 & 25.2 & 22.2 & 0.36 & 8.8 & 178 \\
7 & 8 & 2 & 5 & 6 & 0.86 & 0.91 & 8.4 & 3.8 & 0.12 & 4.5 & 91 \\
$0 \mathrm{~N}$ & 8 & 5 & 8 & 8 & 1.00 & 1.00 & 19.6 & 16.5 & 0.28 & 8.4 & 163 \\
$1 \mathrm{~N}$ & 6 & 3 & 6 & 6 & 1.00 & 1.00 & 12.6 & 7.8 & 0.18 & 6.2 & 118 \\
$2 \mathrm{~N}$ & 7 & 3 & 6 & 6 & 0.87 & 0.87 & 14.7 & 8.3 & 0.21 & 5.7 & 122 \\
$0 \mathrm{~B}$ & 6 & 2 & 4 & 5 & 0.74 & 0.88 & 24.6 & 18.7 & 0.32 & 7.6 & 145 \\
$1 \mathrm{~B}$ & 7 & 4 & 6 & 7 & 0.91 & 1.00 & 20.8 & 19.8 & 0.27 & 9.5 & 125 \\
$2 \mathrm{~B}$ & 6 & 3 & 5 & 6 & 0.84 & 1.00 & 19.3 & 14.4 & 0.25 & 7.5 & 109 \\
\hline
\end{tabular}

Note. -

1. Run ID. Runs with N start with a NFW halo profile, runs with B start with an initial bulge.

2. Total number of black holes formed.

3. Number of black holes within $250 \mathrm{pc}$ of the center at the end of the run (1Gyr).

4. Number of black holes within $500 \mathrm{pc}$ at the end.

5. Number of black holes within $1 \mathrm{kpc}$ at the end.

6. Fractional mass of all black holes that have reached the central 500 pc.

7. Same for the central $1000 \mathrm{pc}$.

8. Bulge mass (measured as in BEE07)

9. Final black hole mass within $500 \mathrm{pc}$

10. Final bulge-to-total mass ratio, not including dark matter in the total.

10. Final nuclear BH to bulge mass ratio, assuming that the IMBHs that reach 500 pc eventually merge.

11. Bulge central line-of-sight velocity dispersion averaged from 50 projections uniformly distributed over the sine of the inclination angle. 


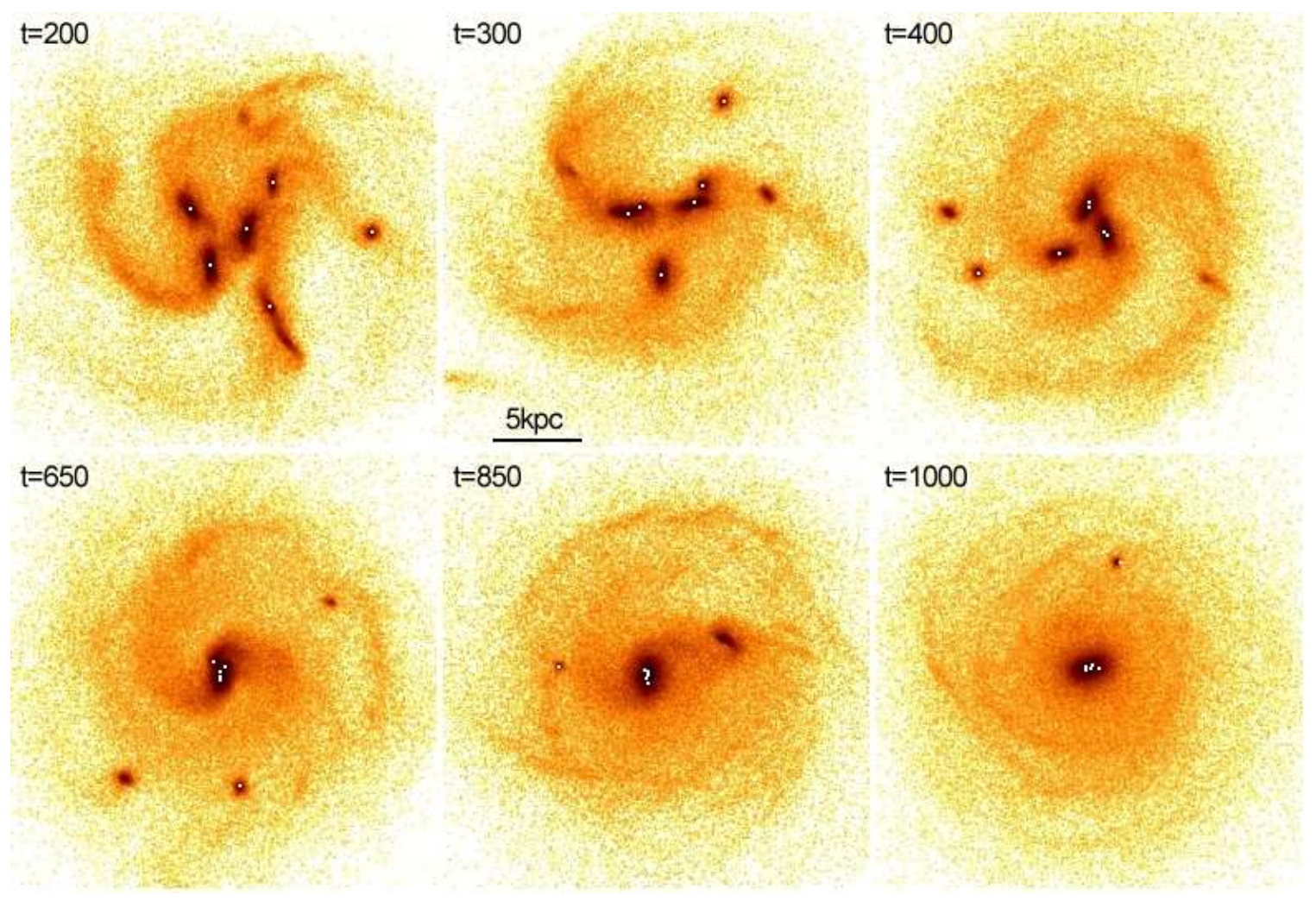

Fig. 1.- Face-on snapshots of the disk mass density (gas and stars) for run 0. White dots correspond to the positions of $\mathrm{BH}$ particles added at the density peak of each clump when the mass fraction in the clumps reaches its maximum. Time is in Myr. The BHs reach the center of the galaxy as a result of clump interactions. 


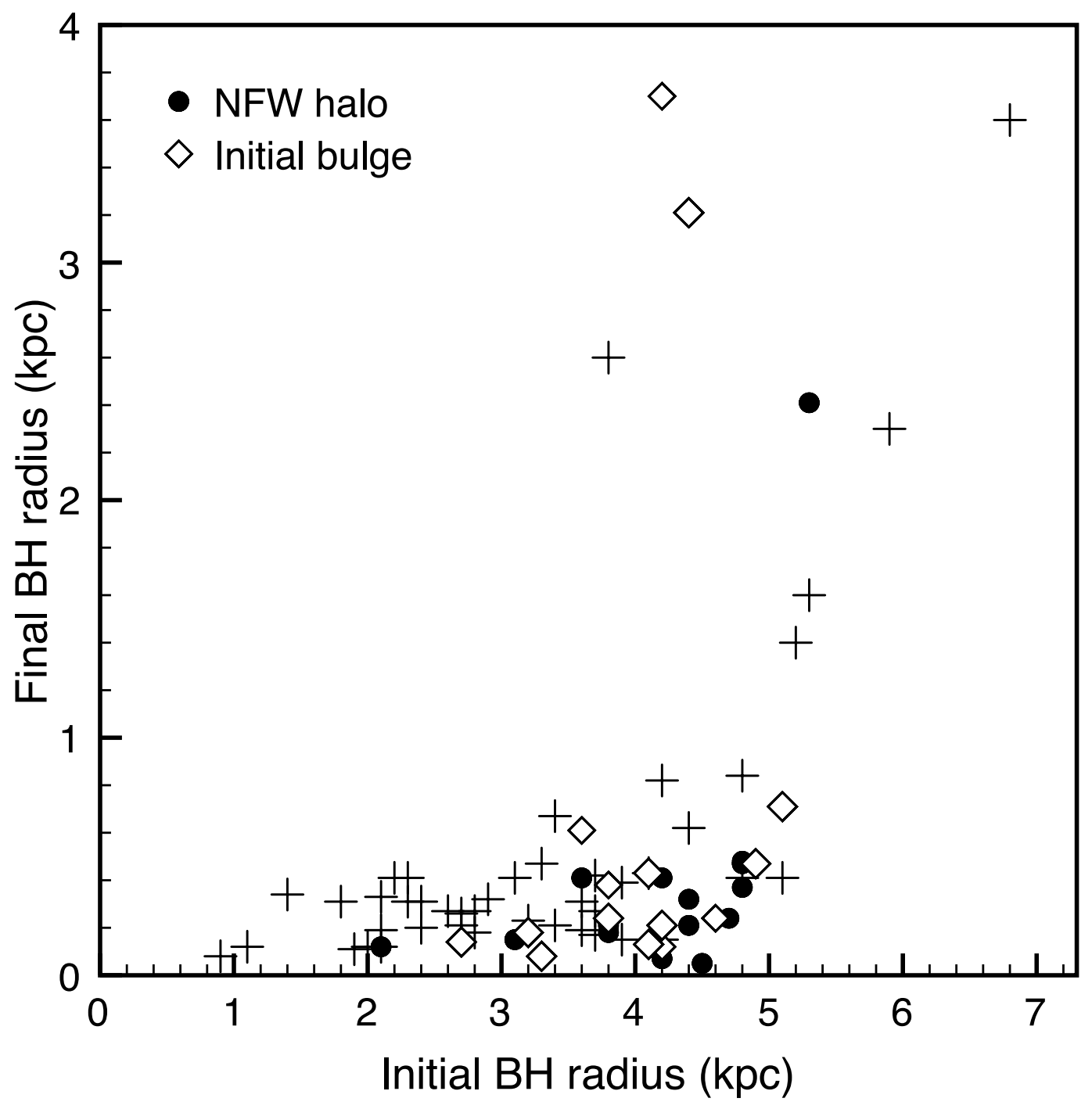

Fig. 2.- Initial and final radius for each black hole particle for the whole model sample. The initial radius is when the $\mathrm{BH}$ particle is created, the final radius is after 1 Gyr. Plus signs correspond to runs 0 to 7 , circles to runs $0 \mathrm{~N}, 1 \mathrm{~N}$ and $2 \mathrm{~N}$ (NFW halo) and diamonds to runs $0 \mathrm{~B}, 1 \mathrm{~B}$ and $2 \mathrm{~B}$ (models with an intial bulge). BHs that begin further out in the disk are less likely to reach the center. 


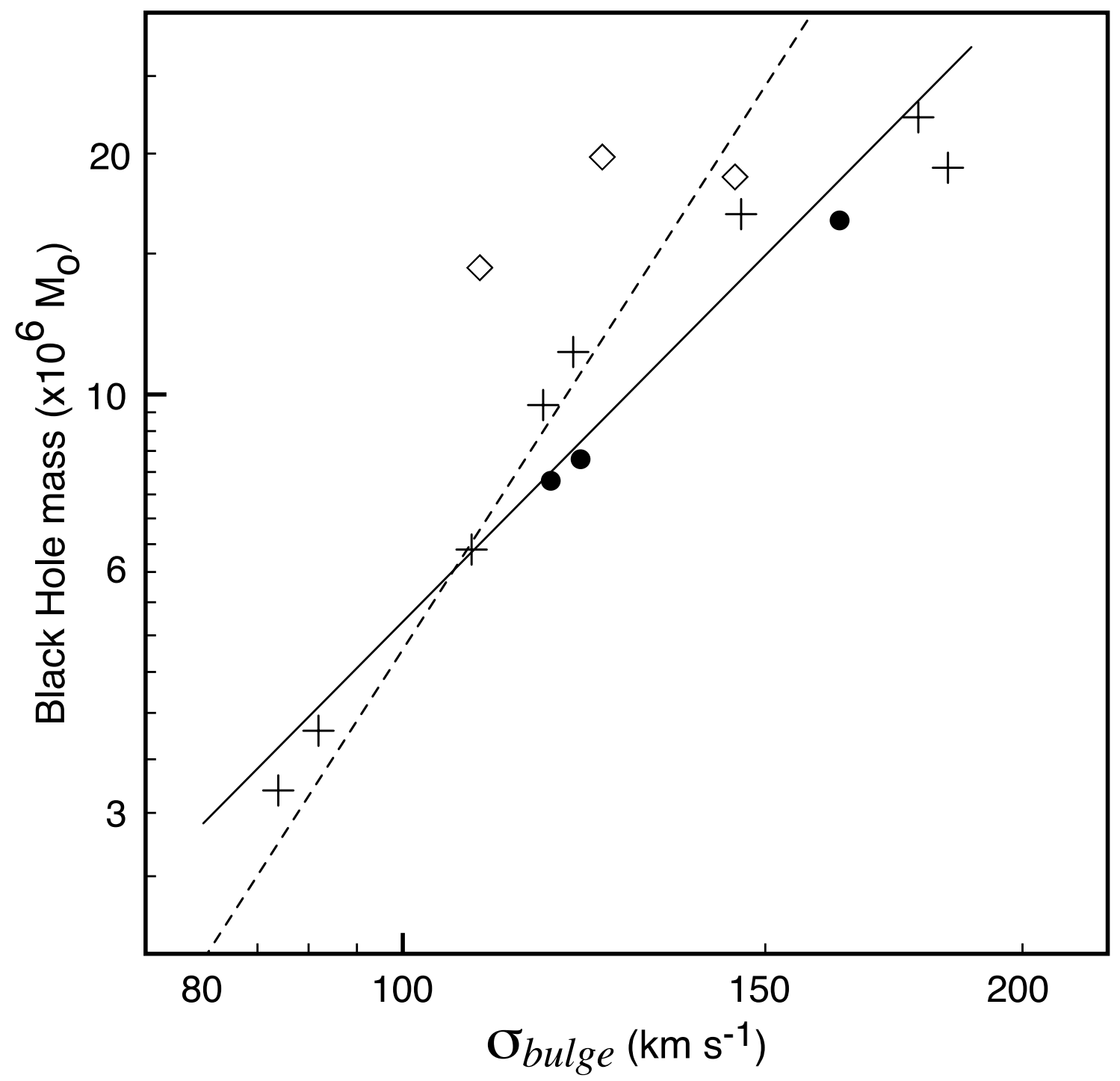

Fig. 3.- The central BH mass (IMBH mass migrated within $500 \mathrm{pc}$ ) is plotted versus the line-of-sight velocity dispersion of the bulge, which is an average over 50 projections uniformly distributed over the sine of the inclination angle. Each point is a different run, with the same symbols as Figure 2. The dashed line has a slope of 4, similar to the observations, and the solid line has a slope of 2 for comparison. 